\begin{tabular}{ll}
\hline \hline MINING AND METALLURGY INSTITUTE BOR & ISSN: 2334-8836 \\
& UDK: 622 \\
\hline \hline
\end{tabular}

Srđana Magdalinović, Ivana Jovanović, Vesna M. Marjanović ${ }^{*}$,Zoran Markovic ${ }^{* *}$

\title{
THE EFFECT OF GRINDING FINENESS ON THE RESULTS OF FLOTATION CONCENTRATION OF ORE FROM THE COPPER DEPOSIT VELIKI KRIVELJ ${ }^{* * *}$
}

\begin{abstract}
One of the influential parameters on the process of flotation concentration is a coarseness of particles and openness of mineral resources. In the process of flotation concentration of ore from the deposit Veliki Krivelj, the grinding fineness since the opening of the mine in 1982 has been maintained at values around 58\% -0.074 mm. Since then, the operation descended into the deeper parts of deposit, the copper content decreased in the ore and it was assumed that the optimal fineness of ore grinding was changed. This paper presents the results of testing the fineness of grin-ding on recovery and copper content of the basic copper concentrate and the impact of regrinding on recovery and copper content in the purified copper concentrate on a sample of ore from the deposits Veliki Krivelj. The laboratory experiments flotation concentration were carried out, the balances of concentration were made and it is concluded that finer grinding is necessary for better concentration. The optimal regrinding degree of basic concentrate was also determined.
\end{abstract}

Keywords: flotation, optimal grinding fineness, copper ore

\section{INTRODUCTION}

The Copper Mine Veliki Krivelj was put into operation in 1982 (Mitrovic Z., Jovanovic R., 2007). The copper deposit Veliki Krivelj belongs to the porphyry deposits of large scale. It is estimated that the balance reserves of copper ore in this deposit are in cut-off grade of $0.15 \% \mathrm{Cu}$, categories $\mathrm{B}+\mathrm{C} 1$ $474,291,085 \mathrm{t}$ with $1,533,821 \mathrm{t}$ of copper (Bugarin M., Maksimović M., Ljubojev V., 2012).

Flotation concentration is a process of separation that takes place due to differences

in physical and chemical properties of mineral surfaces (Barry WA, Napier-Munn, T., 2006). It is mainly used for concentration of metallic ores, cleaning of solid fuel or enrichment of non-metallic minerals, but it can be also applied for separation of solids from liquids or non-mineral particles from each other). Grains of various sizes are not just flotablen even though they have the same composition of mineral surface (Gaudin A. M., 1957). Grinding has a significant impact on technological results (Bulatović S. M., 2007).

\footnotetext{
${ }^{*}$ Mining and Metallurgy Institute Bor, srdjana.magdalinovic@irmbor.co.rs

${ }^{* *}$ University of Belgrade, Technical Faculty Bor

*** This paper is a part of the technological researching Project No. TR33023 "Development the Technologies of Flotation Processing the Copper Ore and Precious Metals for Realization Better Technological Results", funded by the Ministry of Education, Science and Technological Development of the Republic of Serbia.
} 
Optimal grinding fineness of the ore from the Veliki Krivelj deposit was determined in the stage of laboratory testing that preceded the design of site and opening of the mine. Today, 30 years since the opening of the mine, the grinding fineness in the site is maintained as at the beginning of industrial processing at the level of about $58 \%$ $0.074 \mathrm{~mm}$. Some of the technological parameters have been retained to this day, although the exploitation went down into the deeper parts of the ore body, and the copper content is reduced in the ore. It is supposed that some other features essential to the process flotation concentration primarily were changed, primarily the grindability and optimum grinding fineness.

The authors of the paper have considered that it is important to determine the effect of grinding fineness on technological results in the basic flotation, and also the effect of regrinding the primary concentrate on technological results in the purification the basic concentrate on a sample of ore from the deposit Krivelj, which is currently being processed. In the Flotation Plant Veliki Krivelj, regrinding of basic copper concentrate goes to a fineness of $85-90 \%-0.074 \mathrm{~mm}$. Regrinding of the basic copper concentrate is also an important technological process that can contribute to increasing the recovery and copper content in the purified concentrates. Taking into account the estimated reserves of this deposit and the trend of copper value on the world market, any increase in technological results would be significant.

A sample of ore from Veliki Krivelj for testing was taken from production, from a line in front of the rod mill in the Flotation Plant Veliki Krivelj in November 2012.

\section{EXPERIMENTAL PART}

\section{Characterization of sample}

In the Laboratory for mineral processing of the Mining and Metallurgy Institute, a sample is marked VK (Veliki Krivelj), shortened and ground to a size $-3.35 \mathrm{~mm}$. Samples were excluded for chemical analysis and experiments of flotation concentration. Chemical analysis of the sample VK was carried out on three independent samples on copper and sulfur. Table 1 shows the results of chemical analysis. Table 2 presents some characteristic sizes for the sample VK.

Table 1 Chemical analysis of the sample VK

\begin{tabular}{|c|c|c|c|c|c|}
\hline \multicolumn{2}{|c|}{ First sample } & \multicolumn{2}{c|}{ Second sample } & \multicolumn{2}{c|}{ Third sample } \\
\hline $\begin{array}{c}\text { Chemical ele- } \\
\text { ment }\end{array}$ & Content & $\begin{array}{c}\text { Chemical } \\
\text { element }\end{array}$ & Content & $\begin{array}{c}\text { Chemical } \\
\text { element }\end{array}$ & Content \\
\hline Cu & $0.18 \%$ & $\mathbf{C u}$ & $0.24 \%$ & $\mathbf{C u}$ & $0.22 \%$ \\
\hline S & $3.47 \%$ & $\mathbf{S}$ & $2.51 \%$ & $\mathbf{S}$ & $3.04 \%$ \\
\hline
\end{tabular}

Table 2 Characteristic sizes for the sample VK

\begin{tabular}{|l|c|}
\hline Density & $2,820 \mathrm{~kg} / \mathrm{m}^{3}$ \\
\hline Natural pH & 7.45 \\
\hline
\end{tabular}

\section{Grinding}

An ellipsoidal ball mill with a capacity of 15.21 with batch mass of balls at the start of testing of $13.34 \mathrm{~kg}$ was used for testing the grinding. For each grinding experiment, $820 \mathrm{~g}$ of sample was taken.

\section{Grinding kinetics}

For testing the grinding kinetics, the sample was ground with a solid content of $70 \%$, for a period of $3,5,7$ and 9 minutes. Grinding product is sieved on a series of sieves and the grain size analysis results are shown in Table 3. 
Table 3 Grain size distribution of grinding products for various grinding times

\begin{tabular}{|c|c|c|c|c|c|c|c|c|c|c|c|c|}
\hline $\begin{array}{c}\text { Mesh size, } \\
\mathbf{m m}\end{array}$ & \multicolumn{3}{|c|}{$\mathbf{3}$ min } & \multicolumn{3}{c|}{$\mathbf{5}$ min } & \multicolumn{3}{c|}{7 min } & \multicolumn{3}{c|}{ 9 min } \\
\cline { 2 - 13 } \\
$\mathbf{m \%}$ & $\mathbf{D} \%$ & $\mathbf{R} \%$ & $\mathbf{m} \%$ & $\mathbf{D} \%$ & $\mathbf{R} \%$ & $\mathbf{m} \%$ & $\mathbf{D} \%$ & $\mathbf{R} \%$ & $\mathbf{m} \%$ & $\mathbf{D} \%$ & $\mathbf{R} \%$ \\
\hline$-0,600+0,425$ & 2,0 & 100,0 & 2,0 & & & & & & & & & \\
\hline$-0,425+0,300$ & 6,4 & 98,0 & 8,4 & & & & & & & & & \\
\hline$-0,300+0,212$ & 10,0 & 91,6 & 18,4 & 3,4 & 100,0 & 3,4 & & & & & & \\
\hline$-0,212+0,150$ & 11,4 & 81,6 & 29,8 & 7,6 & 96,6 & 11,0 & 4,8 & 100,0 & 4,80 & 2,6 & 100,0 & 2,6 \\
\hline$-0,150+0,106$ & 10,0 & 70,2 & 39,8 & 10,4 & 89,0 & 21,4 & 9,4 & 95,2 & 14,2 & 5,8 & 97,4 & 8,4 \\
\hline$-0,106+0,075$ & 6,8 & 60,2 & 46,6 & 10,6 & 78,6 & 32,0 & 11,2 & 85,8 & 25,4 & 10,6 & 91,6 & 19,0 \\
\hline$-0,075+0,053$ & 5,4 & 53,4 & 52,0 & 8,4 & 68,0 & 40,4 & 9,8 & 74,6 & 35,2 & 10,4 & 81,0 & 29,4 \\
\hline$-0,053+0,038$ & 6,0 & 48,0 & 58,0 & 7,0 & 59,6 & 47,4 & 8,6 & 64,8 & 43,8 & 10,0 & 70,6 & 39,4 \\
\hline$-0,038+0$ & 42,0 & 42,0 & 100,0 & 52,6 & 52,6 & 100,0 & 56,2 & 56,2 & 100,0 & 60,6 & 60,6 & 100,0 \\
\hline
\end{tabular}

Figure 1 presents the grinding kinetics.

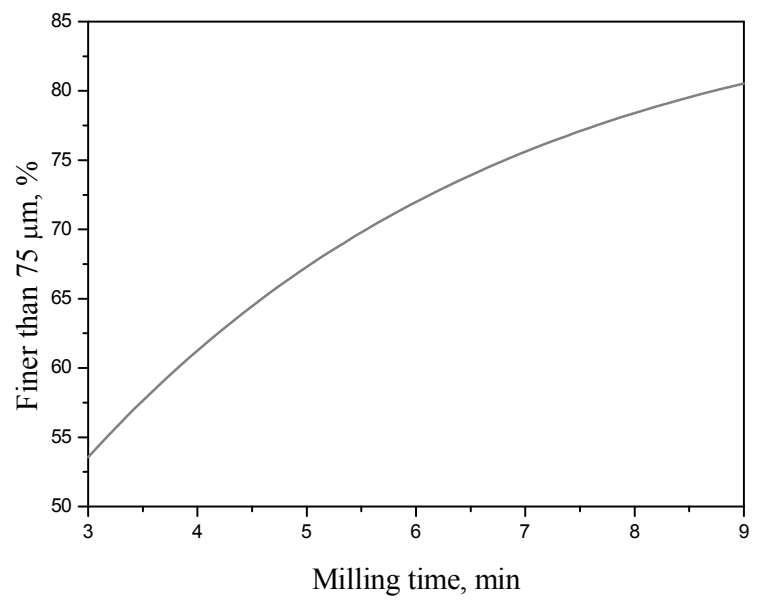

Figure 1 Grinding kinetics

Testing the effect of grinding fineness on the results of basic flotation

The effect on grinding fineness on the basic flotation results was tested by grin-

flotation machine, in a chamber volume 2.41 with $820 \mathrm{~g}$ of sample. Concentration ding, and then flotation in a Denver D-12 products were analyzed chemically, and 
then the concentration balance was calculated for each experiment.

A series of experiments was done that included the grinding fineness of $55-80 \%$ $-0.075 \mathrm{~mm}$. Experiments were carried out according to the scheme shown in Figure
2. The obtained results of concentration are shown in Table 4. Based on the results in Table 4, dependence of copper recovery and content in the concentrate was carried out on grinding fineness, and it is shown in Figure 3.

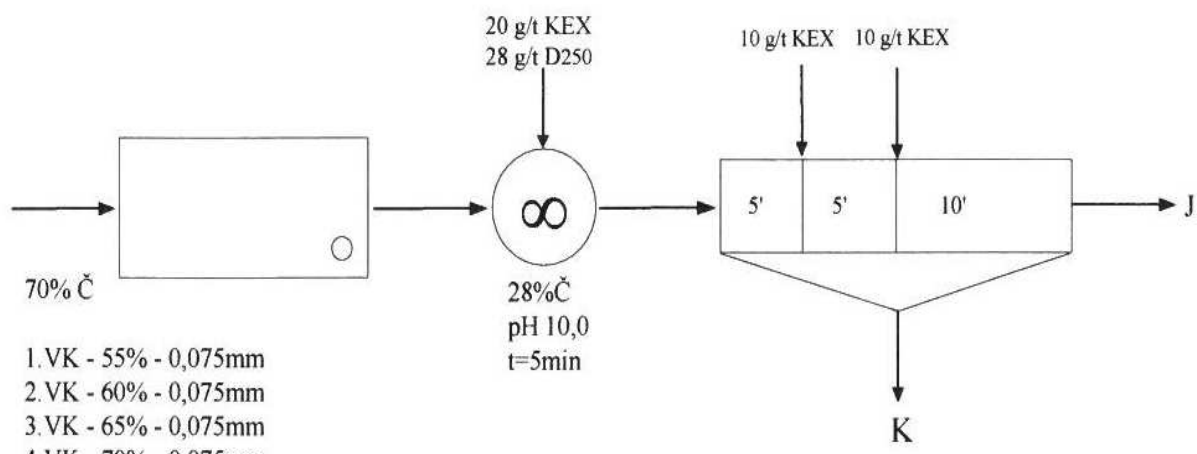

4.VK $-70 \%-0,075 \mathrm{~mm}$

5.VK $-75 \%-0,075 \mathrm{~mm}$

6. VK $-80 \%-0,075 \mathrm{~mm}$

Figure 2 Scheme of testing the effect of grinding fineness

Table 4 Results of the basic flotation of copper minerals

\begin{tabular}{|c|c|c|c|c|c|}
\hline \multirow{2}{*}{ PRODUCT } & \multirow{2}{*}{$\begin{array}{c}\text { MASS } \\
\text { m, } \%\end{array}$} & \multicolumn{2}{|c|}{ CONTENT } & \multicolumn{2}{|c|}{ DISTRIBUTION } \\
\hline & & $\mathrm{Cu}, \%$ & $\mathrm{~S}, \%$ & $\mathrm{Cu}, \%$ & $\mathrm{~S}, \%$ \\
\hline \multicolumn{6}{|c|}{ Sample 1. VK 55\% - 0,075 mm } \\
\hline $\mathrm{U}$ & 100,00 & 0,1843 & 3,5096 & 100,00 & 100,00 \\
\hline $\mathrm{K}$ & 10,49 & 1,50 & 31,75 & 85,40 & 94,90 \\
\hline $\mathrm{J}$ & 89,51 & 0,03 & 0,2 & 14,60 & 5,10 \\
\hline \multicolumn{6}{|c|}{ Sample 2. VK 60\% - 0,075 mm } \\
\hline $\mathrm{U}$ & 100,00 & 0,2169 & 2,9674 & 100,00 & 100,00 \\
\hline $\mathrm{K}$ & 10,93 & 1,74 & 25,52 & 87,69 & 94,00 \\
\hline $\mathrm{J}$ & 89,07 & 0,03 & 0,2 & 12,31 & 6,00 \\
\hline \multicolumn{6}{|c|}{ Sample 3. VK 65\% - 0,075 mm } \\
\hline $\mathrm{U}$ & 100,00 & 0,2154 & 3,1449 & 100,00 & 100,00 \\
\hline $\mathrm{K}$ & 11,99 & 1,65 & 23,44 & 91,83 & 89,37 \\
\hline $\mathrm{J}$ & 88,01 & 0,02 & 0,38 & 8,17 & 10,63 \\
\hline
\end{tabular}




\begin{tabular}{|c|c|c|c|c|c|}
\hline \multicolumn{7}{|c|}{ Sample 4. VK 70\%-0,075 mm } \\
\hline $\mathrm{U}$ & 100,00 & 0,2123 & 2,8124 & 100,00 & 100,00 \\
\hline $\mathrm{K}$ & 11,72 & 1,66 & 22,49 & 91,66 & 93,72 \\
\hline $\mathrm{J}$ & 88,28 & 0,02 & 0,2 & 8,34 & 6,28 \\
\hline \multicolumn{7}{|c|}{ Sample $5 . V K 75 \%-0,075 \mathrm{~mm}$} \\
\hline $\mathrm{U}$ & 100,00 & 0,2175 & 3,1000 & 100,00 & 100,00 \\
\hline $\mathrm{K}$ & 11,90 & 1,68 & 24,57 & 91,91 & 94,32 \\
\hline $\mathrm{J}$ & 88,10 & 0,02 & 0,2 & 8,09 & 5,68 \\
\hline \multicolumn{7}{|c|}{ Sample $6 . V K 80 \%-0,075 \mathrm{~mm}$} \\
\hline $\mathrm{U}$ & 100,00 & 0,2319 & 3,6072 & 100,00 & 100,00 \\
\hline $\mathrm{K}$ & 12,61 & 1,70 & 27,22 & 92,45 & 95,15 \\
\hline $\mathrm{J}$ & 87,39 & 0,02 & 0,2 & 7,55 & 4,85 \\
\hline
\end{tabular}

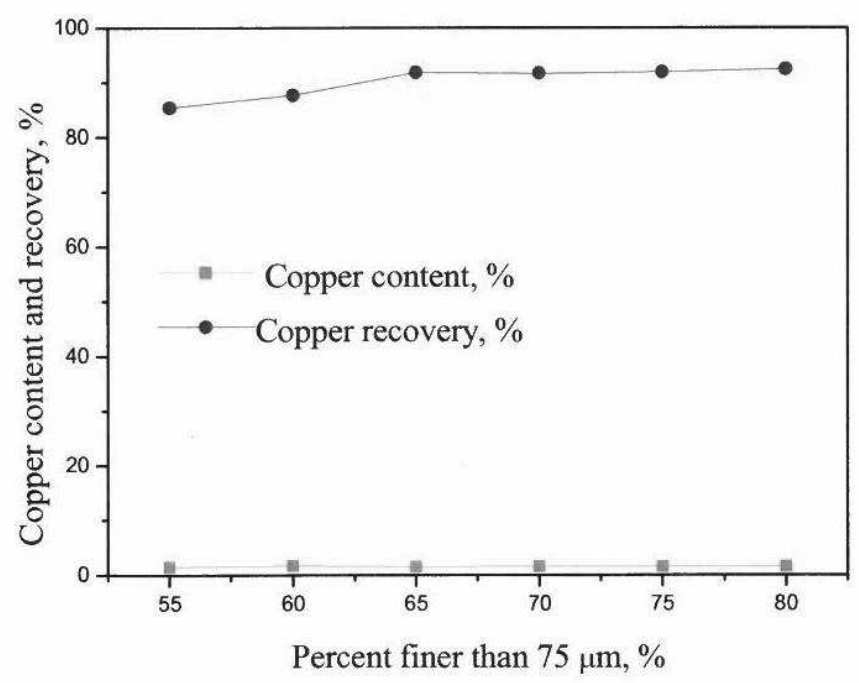

Figure 3 Dependence of copper recovery and content in the basic concentrate on grinding fineness

\section{Regrinding}

An elliptical ball mill, capacity $15.2 \mathrm{~L}$, with a mass batch of balls of $3,755 \mathrm{~kg}$ at the beginning of testing, was used for testing of regrinding. For each experiment of regrinding, it was necessary to grind the four samples of $820 \mathrm{~g}$ mass. After that, the two basic 
flotation processes were carried out (where the mass of samples in each basic flotation was $1.640 \mathrm{~g}$ ) in a Denver flotation machine D-12 in a chamber, volume of 4.61 , under the same conditions, and them the basic regrinding concentrate was obtained in a ball mill at different time intervals. The regrinding product was once purified in a flotation machine Denver D-12 in the chamber of 2.4 1 , and the concentration products were che- mically analyzed, after which the concentration balance was calculated.

\section{Testing the regrinding kinetics}

A scheme, according to which the regrinding kinetics was tested, is shown in Figure 4 . The results of grain size analysis of regrinding products are shown in Table 6 , and regrinding kinetics in Figure 5.

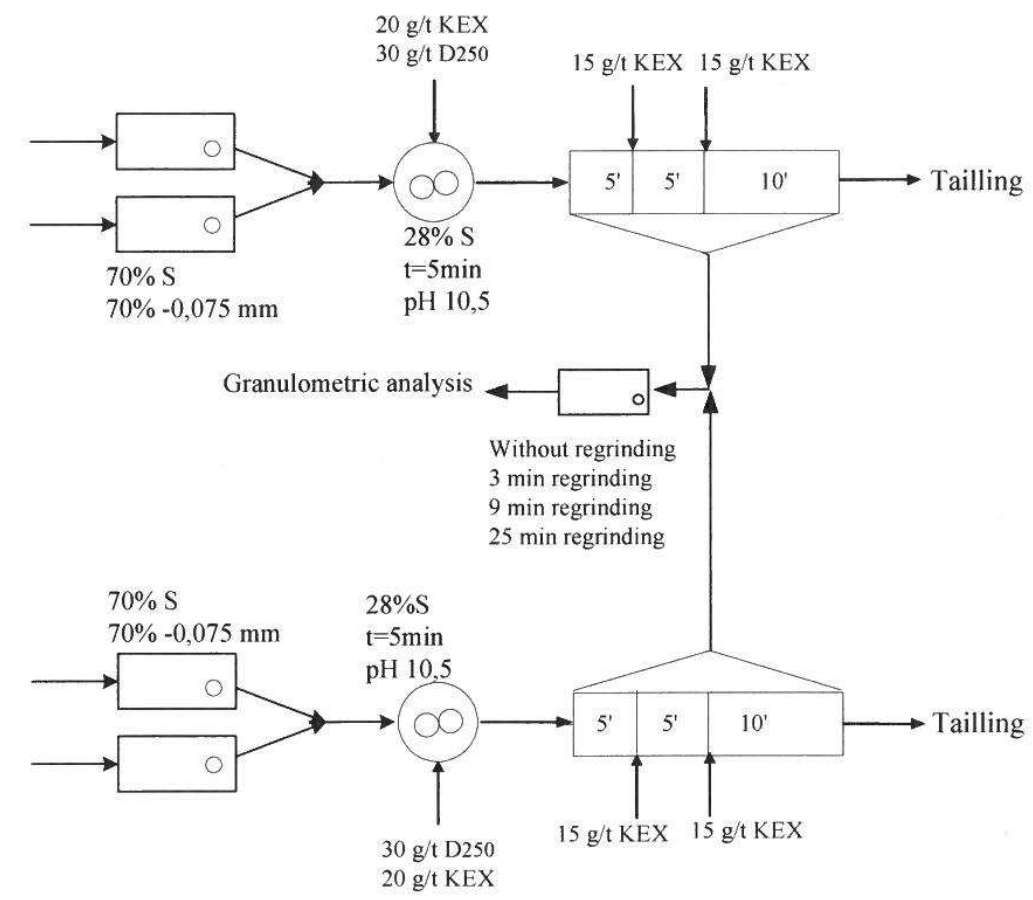

Figure 4 Scheme of testing the regrinding kinetics

Table 6 Grain size distribution of regrinding products for various regrinding times

\begin{tabular}{|c|c|c|c|c|c|c|c|c|}
\hline \multirow{2}{*}{ Mesh size, $\mathbf{~ m m}$} & \multicolumn{2}{|c|}{ Without regrinding } & \multicolumn{2}{c|}{ 3 min. } & \multicolumn{2}{c|}{ 9 min. } & \multicolumn{2}{c|}{ 25 min. } \\
\cline { 2 - 10 } & $\mathbf{m}$ \% & D\% & $\mathbf{m}$ \% & D \% & m \% & D \% & m \% & D \% \\
\hline$-0.300+0.212$ & 2.00 & 100.00 & & & & & & \\
\hline$-0.212+0.150$ & 6.67 & 98.00 & 2.57 & 100.00 & & & & \\
\hline$-0.150+0.106$ & 11.89 & 91.33 & 7.38 & 97.43 & 3.32 & 100.00 & 0.86 & 100.00 \\
\hline$-0.106+0.075$ & 11.22 & 79.44 & 10.81 & 90.05 & 7.00 & 96.68 & 3.86 & 99.14 \\
\hline$-0.075+0.053$ & 8.22 & 68.22 & 9.81 & 79.24 & 9.84 & 89.68 & 6.72 & 95.28 \\
\hline$-0.053+0.038$ & 7.00 & 60.00 & 8.62 & 69.43 & 9.84 & 79.84 & 8.72 & 88.56 \\
\hline$-0.038+0$ & 53.00 & 53.00 & 60.81 & 60.81 & 70.00 & 70.00 & 79.84 & 79.84 \\
\hline
\end{tabular}




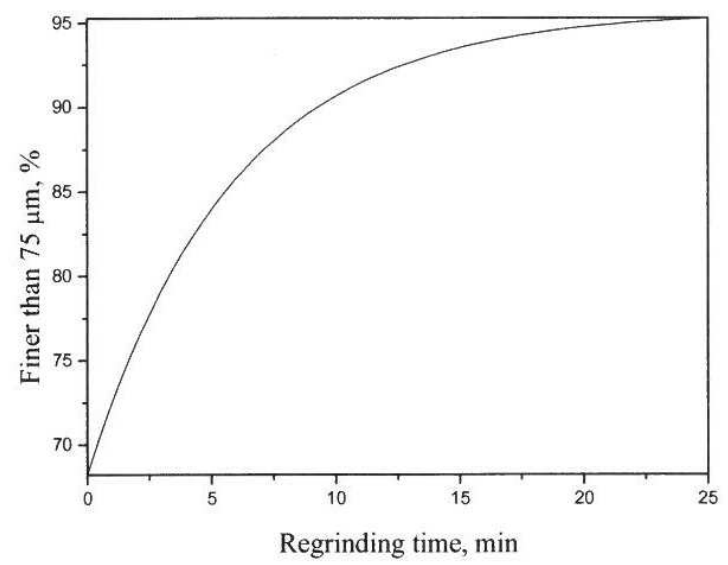

Figure 5 Regrinding kinetics

The effect of regrinding fineness on the results of flotation concentration

The effect of grinding fineness on the results of flotation concentration was tested in four experiments, and the scheme according to which the experiments were carried out is shown in Figure 6. The results of regrinding effect on purification the copper concentrate are shown in Table 7, and Figure 7 shows the dependence of copper recovery and content in the purified copper concentrate from regrinding fineness.

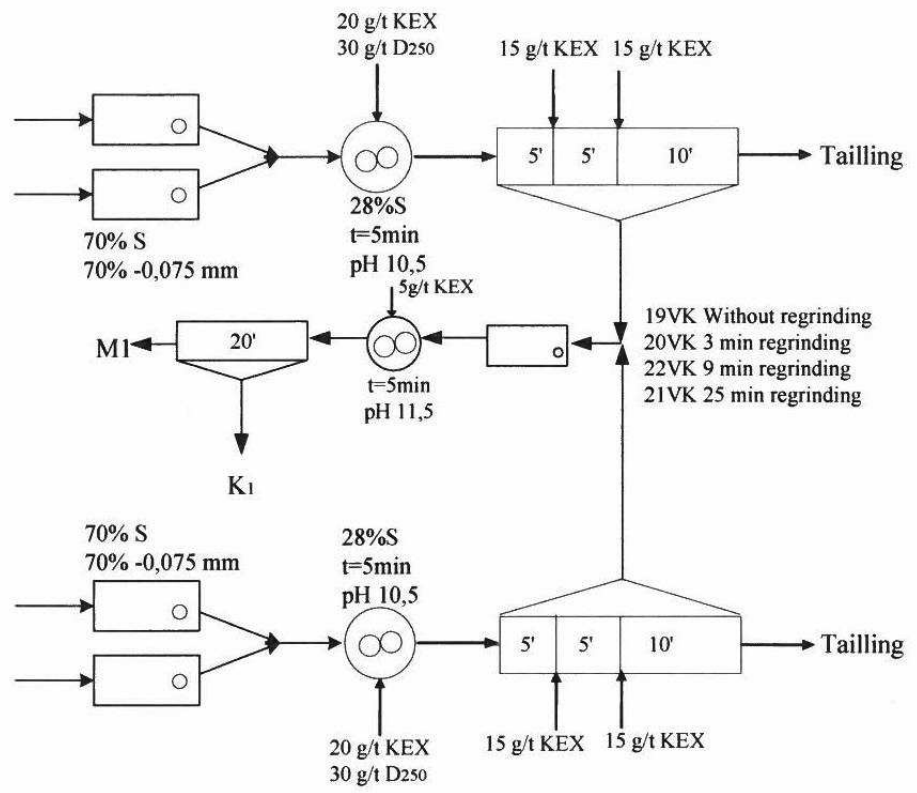

Figure 6 Scheme of testing the grinding fineness on the flotation results 
Table 7 The effect of regrinding fineness on the results of purification the copper concentrate

\begin{tabular}{|c|c|c|c|c|c|c|c|c|c|}
\hline \multirow[b]{2}{*}{ PROD. } & \multirow{2}{*}{$\begin{array}{c}\text { MASS } \\
\text { m, \% }\end{array}$} & \multicolumn{4}{|c|}{ CONTENT } & \multicolumn{4}{|c|}{ DISTRIBUTION } \\
\hline & & $\begin{array}{c}\mathrm{Cu}, \\
\%\end{array}$ & $\mathrm{~S}, \%$ & $\begin{array}{c}\mathbf{A u}, \\
\mathrm{g} / \mathrm{t}\end{array}$ & $\begin{array}{c}\mathbf{A g}, \\
\mathbf{g} / \mathbf{t}\end{array}$ & $\begin{array}{c}\mathbf{C u}, \\
\%\end{array}$ & $\mathrm{~S}, \%$ & $\begin{array}{c}\mathbf{A u}, \\
\mathrm{g} / \mathrm{t}\end{array}$ & $\begin{array}{l}\text { Ag, } \\
\text { g/t }\end{array}$ \\
\hline \multicolumn{10}{|c|}{ Sample $19 \mathrm{VK}-68.22 \%-0.075 \mathrm{~mm}$; without regrinding } \\
\hline $\mathrm{U}$ & 100.00 & 0.1855 & 2.8081 & 0.073 & 0.80 & 100.00 & 100.00 & 100.00 & 100.00 \\
\hline $\mathrm{J}$ & 90.36 & 0.010 & 0.27 & 0.016 & 0.713 & 4.85 & 8.69 & 19.25 & 80.58 \\
\hline Ko & 9.64 & 1.83 & 26.60 & 0.612 & 1.612 & 95.15 & 91.31 & 80.75 & 19.42 \\
\hline $\mathrm{M}_{1}$ & 7.49 & 0.14 & 26.89 & 0.5 & 1.50 & 5.66 & 71.71 & 51.30 & 14.04 \\
\hline $\mathrm{K}_{1}$ & 2.15 & 7.72 & 25.60 & 1.0 & 2.00 & 89.49 & 19.60 & 29.45 & 5.38 \\
\hline \multicolumn{10}{|c|}{ Sample 20 VK - $79.24 \%-0.075 \mathrm{~mm} ; 3$ minutes } \\
\hline $\mathrm{U}$ & 100.00 & 0.1864 & 3.1504 & 0.080 & 0.80 & 100.00 & 100.00 & 100.00 & 100.00 \\
\hline $\mathrm{J}$ & 89.44 & 0.012 & 0.2 & 0.019 & 0.671 & 5.74 & 5.68 & 21.24 & 75.05 \\
\hline Ko & 10.56 & 1.66 & 28.14 & 0.597 & 1.89 & 94.26 & 94.32 & 78.86 & 24.95 \\
\hline $\mathrm{M}_{1}$ & 8.55 & 0.06 & 28.99 & 0.384 & 1.70 & 2.74 & 78.68 & 41.07 & 18.17 \\
\hline $\mathrm{K}_{1}$ & 2.01 & 8.49 & 24.52 & 1.50 & 2.70 & 91.52 & 15.65 & 37.69 & 6.78 \\
\hline \multicolumn{10}{|c|}{ Sample 22 VK- $89.68 \%-0.075 \mathrm{~mm} ; 9$ minutes } \\
\hline $\mathrm{U}$ & 100.00 & 0.1988 & 3.0517 & 0.073 & 0.80 & 100.00 & 100.00 & 100.00 & 100.00 \\
\hline $\mathrm{J}$ & 90.69 & 0.012 & 0.36 & 0.020 & 0.68 & 5.48 & 10.70 & 24.85 & 77.09 \\
\hline Ko & 9.31 & 2.02 & 29.27 & 0.589 & 1.969 & 94.52 & 89.30 & 75.15 & 22.91 \\
\hline $\mathrm{M}_{1}$ & 7.78 & 0.14 & 29.89 & 0.414 & 1.80 & 5.48 & 76.20 & 44.16 & 17.50 \\
\hline $\mathrm{K}_{1}$ & 1.53 & 11.57 & 26.13 & 1.478 & 2.828 & 89.03 & 13.10 & 30.99 & 5.41 \\
\hline \multicolumn{10}{|c|}{ Sample $21-95.28 \%-0.075 \mathrm{~mm} ; 25$ minutes } \\
\hline $\mathrm{U}$ & 100.00 & 0.1980 & 2.8367 & 0.073 & 0.80 & 100.00 & 100.00 & 100.00 & 100.00 \\
\hline $\mathrm{J}$ & 90.35 & 0.012 & 0.2 & 0.017 & 0.706 & 5.45 & 6.37 & 21.04 & 79.73 \\
\hline Ko & 9.65 & 1.94 & 27.52 & 0.597 & 1.681 & 94.55 & 93.63 & 78.96 & 20.27 \\
\hline $\mathrm{M}_{1}$ & 8.31 & 0.15 & 27.92 & 0.403 & 1.50 & 6.31 & 81.79 & 45.92 & 15.58 \\
\hline $\mathrm{K}_{1}$ & 1.34 & 13.04 & 25.06 & 1.80 & 2.80 & 88.23 & 11.84 & 33.04 & 4.69 \\
\hline
\end{tabular}

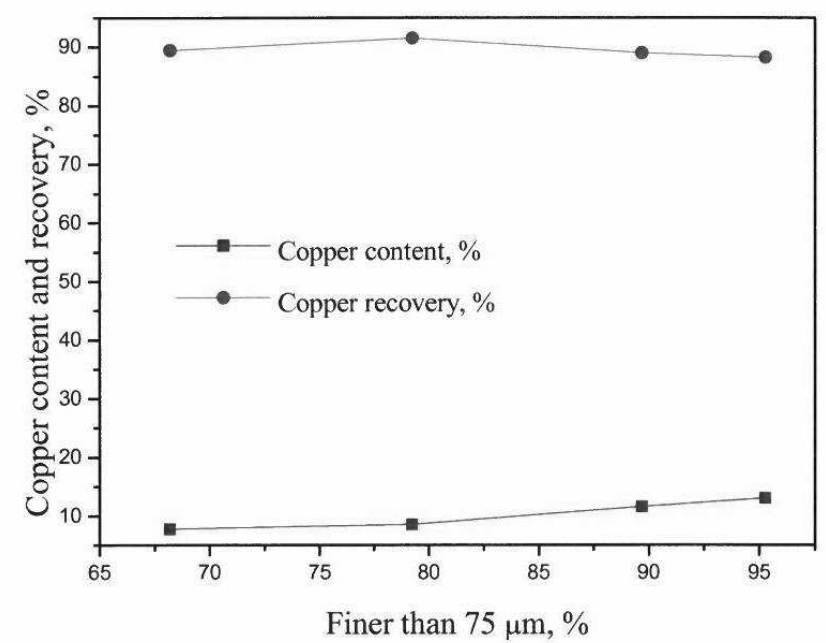

Figure 7 The effect of regrinding fineness on the results of concentration 


\section{CONCLUSION}

Optimal grinding fineness in the process of flotation concentration of the ore from the Veliki Krivelj deposit is determined on the basis of laboratory tests that preceded the design of the site and opening of the mine. Today, almost 30 years since opening of the mine, the grinding fineness in the site is maintained as at the beginning of production. To determine whether this important parameter corresponds to the ore being processed today, testing was carried out on a sample of ore, taken from production. A sample of the ore Veliki Krivelj is marked $\mathrm{VK}$, shortened and ground to a size -3.35 $\mathrm{mm}$. Samples were excluded for chemical analysis and flotation concentration experiments and the tests were carried out on them.

The mean content of the most important elements in the sample, calculated from the chemical analysis was: $0.2133 \% \mathrm{Cu}$; $3.0067 \% \mathrm{~S} ; 0.0733 \mathrm{~g} / \mathrm{t} \mathrm{Au} ; 0.5667 \mathrm{~g} / \mathrm{t} \mathrm{Ag}$. The mean content of the most important elements in the sample VK, when it is calculated over the concentration products of all experiments: $0.2095 \% \mathrm{Cu} ; 3.1998 \% \mathrm{~S}$; $0.0709 \mathrm{~g} / \mathrm{t} \mathrm{Au} ; 0.6614 \mathrm{~g} / \mathrm{t} \mathrm{Ag}$. The density of the sample VK was $2,820 \mathrm{~kg} / \mathrm{m}^{3}$; the natural $\mathrm{pH}$ 7.20. During laboratory technological tests on a sample VK, the following conclusions were made:

- Minimum grinding fineness to be achieved on a sample VK, in terms of technological results is $65 \%$ $-0.075 \mathrm{~mm}$,

- Regrinding of the basic concentrate has little effect on copper recovery in concentrate from the first purification, but copper content in concentrate increases. The results indicate that the regrinding of basic concentrate is preferably up to $80-90 \%-0,075 \mathrm{~mm}$ do not go over $90 \%-0.075 \mathrm{~mm}$ because, in this case, the copper recovery decreases.

The results of laboratory experiments have shown that minimum grinding fineness to be achieved on a sample VK is $65 \%-0.075 \mathrm{~mm}$. This increases the copper recovery in the basic concentrate for about $4 \%$. Further increasing the grinding fineness would lead to an increase of copper recovery by another $1 \%$.

The authors of testing think that the responsible in RTB Bor should consider the possibility of increasing the grinding fineness of the ore from the deposit Veliki Krivelj, because it will surely have positive financial effects. Complex economic analysis could exactly point out to which fineness should be adopted, because it is a compromise between a few essential items in production:

- increasing the power consumption to finer grinding,

- increasing the amount of copper in concentrate with increasing the grinding fineness, and

- capacity reduction of the mill sections, because it is only possible to realize finer grinding in given conditions.

\section{REFERENCES}

[1] Mitrović Z., Jovanović R., A Hundred Years of the Bor Mining 1903-2003, Sevety Five years of the Basin Flotation Plants 1929-2003., RTB Bor, Megatrend University Belgrade, 2007(in Serbian);

[2] Bugarin M., Maksimović M., Ljubojev V., Copper and Gold Reserves in the Exploratory - Mining Field of RTB Bor; Mining Engineering, Mining and 
Metallurgy Institute Bor, ISSN 14510162, 3/2012, pp. 9-16;

[3] Barry W. A., Napier-Munn T., Wills' Mineral Processing Technology, Elsevier Science \& Technology Books, 2006;
[4] Gaudin A. M., Flotation, McGraw-Hill Book Company, Inc., New York, Toronto, London, 1957.

[5] Bulatovic S. M., Handbook of Flotation Reagents, Volume 1, Elsevier Science \& Technology Books, 2007. 


\begin{tabular}{ll}
\hline \hline INSTITUT ZA RUDARSTVO I METALURGIJU BOR & ISSN: 2334-8836 \\
& UDK: 622 \\
\hline \hline
\end{tabular}

Srđana Magdalinović, Ivana Jovanović, Vesna M. Marjanović ${ }^{*}$,Zoran Markovic ${ }^{* *}$

\section{UTICAJ FINOĆE MLEVENJA NA \\ REZULTATE FLOTACIJSKE KONCENTRACIJE RUDE LEŽIŠTA BAKRA VELIKI KRIVELJ ${ }^{* * *}$}

Izvod

Jedan od uticajnih parametara na proces flotacijske koncentracije je krupnoća čestica i otvorenost mineralne sirovine. U procesu flotacijske koncentracije rude ležišta Veliki Krivelj finoća mlevenja se od otvaranja rudnika 1982. godine održava na vrednosti oko 58\% -0,074 mm. Od tada se sišlo u dublje partije ležišta, opao je sadržaj bakra u rudi pa se pretpostavilo da se promenila i optimalna finoća mlevenja rude. $U$ radu su prikazani rezultati ispitivanja uticaja finoće mlevenja na iskorišćenje $i$ sadržaj bakra u osnovnom koncentratu bakra i uticaj domeljavanja na iskorišćenje i sadržaj bakra u prečišćenom koncentratu bakra na uzorku rude ležišta Veliki Krivelj. Urađeni su laboratorijski eksperimenti flotacijske koncentracije, obračunati bilansi koncentracije i donet je zaključak da je za bolje rezultate koncentracije neophodno finije mlevenje. Takođe je utvrđen optimalni stepen domeljavanja osnovnog koncentrata.

Ključne reči: flotacija, optimalna finoća mlevenja, ruda bakra

\section{UVOD}

Rudnik bakra Veliki Krivelj pušten je u rad 1982. godine (Mitrović Z., Jovanović R., 2007.). Ležište bakra Veliki Krivelj pripada porfirskim ležištima velikih razmera. Procenjuje se da su bilansne rezerve rude bakra u ovom ležištu u graničnom sadržaju od 0,15 \% Cu, kategorije B+C1 474. 291.085 t u kojima ima 1.533.821 t bakra (Bugarin M., Maksimović M., Ljubojev V., 2012.).

Flotacijska koncentracija je proces razdvajanja koji se odvija zahvaljujući razlikama u fizičko hemijskim osobinama mineralnih površina (Barry W. A., NapierMunn T., 2006.). Uglavnom se primenjuje za koncentraciju metaličnih ruda, za čišćenje čvrstih goriva ili za obogaćivanje nemetaličnih minerala, ali može da se primeni i za izdvajanje čvrstih čestica iz tečnosti ili razdvajanje nemineralnih čestica jednih od drugih). Zrna različite krupnoće, nisu jednako flotabilna čak iako imaju isti sastav mineralne površine (Gaudin A. M., 1957.). Mlevenje značajno utiče na tehnološke rezultate (Bulatović S. M., 2007.).

\footnotetext{
* Institut za rudarstvo i metalurgiju Bor, srdjana.magdalinovic@irmbor.co.rs

** Univerzitet u Beogradu, Tehnički Fakultet Bor

*** Ovaj rad je proistekao kao rezultat projekta TR33023 "Razvoj tehnologija flotacijske prerade ruda bakra i plemenitih metala radi postizanja boljih tehnoloških rezultata" finansiranog od strane Ministarstva prosvete, nauke i tehnološkog razvoja Republike Srbije
} 
Optimalna finoća mlevenja na rudi ležišta Veliki Krivelj određena je u fazi laboratorijskih ispitivanja koja su prethodila projektovanju pogona $\mathrm{i}$ otvaranju rudnika. Danas, 30 godina od otvaranja rudnika, finoća mlevenja u pogonu održava se kao i na početku industrijske prerade na nivou oko $58 \% \quad-0,074 \mathrm{~mm}$. Neki od tehnoloških parametara zadržani su do danas, iako se tokom eksploatacije sišlo u dublje partije rudnog ležišta, i smanjio se sadržaj bakra u rudi. Pretpostavlja se da su se promenile i neke druge karakteristike rude bitne za proces flotacijske koncentracije pre svega meljivost i optimalna finoća mlevenja.

Autori rada su smatrali da je bitno utvrditi uticaj finoće mlevenja na tehnološke rezultate u osnovnom flotiranju a takođe i uticaj domeljavanja osnovnog koncentrata na tehnološke rezultate $\mathrm{u}$ prečišćavanju osnovnog koncentrata na uzorku rude ležišta Veliki Krivelj, koja se trenutno prerađuje. U Flotaciji Veliki Krivelj, domeljavanje osnovnog koncentrata bakra ide do finoće od 85 - 90\% -0,074 mm. Domeljavanje osnovnog koncentrata bakra je takođe važan tehnološki postupak koji može da doprinese povećanju iskorišćenja i sadržaja bakra u prečišćenom koncentratu. Imajući u vidu procenjene rezerve $u$ ovom ležištu i trend vrednosti bakra na svetskom tržištu svako povećanje tehnoloških rezultata bilo bi značajno.

Uzorak rude za ispitivanja Veliki Krivelj izuzet je iz proizvodnje, sa trake ispred mlina sa šipkama u pogonu Flotacije Veliki Krivelj u novembru 2012. godine.

\section{EKSPERIMENTALNI DEO}

\section{Karakterizacija uzorka}

U Laboratoriji za PMS, Instituta za rudarstvo i metalurgiju uzorak je označen oznakom VK, skraćen i usitnjen do krupnoće $-3,35 \mathrm{~mm}$. Izuzeti su uzorci za hemijsku analizu i eksperimente flotacijske koncentracije. Hemijska analiza uzorka VK je urađena na tri nezavisna uzorka na bakar i sumpor. U tablici 1 prikazani su rezultati hemijske analize. U tablici 2 prikazane su neke karakteristične veličine za uzorak VK.

Tabela 1. Hemijska analiza uzorka VK

\begin{tabular}{|c|c|c|c|c|c|}
\hline \multicolumn{2}{|c|}{ Prvi uzorak } & \multicolumn{2}{c|}{ Drugi uzorak } & \multicolumn{2}{c|}{ Treći uzorak } \\
\hline $\begin{array}{c}\text { Hemijski } \\
\text { element }\end{array}$ & Sadržaj & $\begin{array}{c}\text { Hemijski } \\
\text { element }\end{array}$ & Sadržaj & $\begin{array}{c}\text { Hemijski } \\
\text { element }\end{array}$ & Sadržaj \\
\hline Cu & $0,18 \%$ & $\mathbf{C u}$ & $0,24 \%$ & $\mathbf{C u}$ & $0,22 \%$ \\
\hline $\mathbf{S}$ & $3,47 \%$ & $\mathrm{~S}$ & $2,51 \%$ & $\mathrm{~S}$ & $3,04 \%$ \\
\hline
\end{tabular}

Tabela 2. Karakteristične veličine za uzorak VK

\begin{tabular}{|l|c|}
\hline Gustina & $2.820 \mathrm{~kg} / \mathrm{m}^{3}$ \\
\hline Prirodna pH & 7,45 \\
\hline
\end{tabular}

\section{Mlevenje}

Za ispitivanje mlevenja, korišćen je laboratorijski elipsoidni mlin sa kuglama zapremine 15,2 1 sa masom šarže kugli na početku ispitivanja od $13,34 \mathrm{~kg}$. Za svaki eksperiment mlevenja uzeto je $820 \mathrm{~g}$ uzorka.

\section{Kinetika mlevenja}

Za ispitivanje kinetike mlevenja, uzorak je mleven pri sadržaju čvrstog od $70 \%$, u trajanju od 3, 5, 7 i 9 minuta. Proizvod mlevenja je prosejan na seriji sita, a rezultati granulometrijske analize su prikazani u tabeli 3 . 
Tabela 3. Granulometrijski sastav proizvoda mlevenja za različita vremena mlevenja

\begin{tabular}{|c|c|c|c|c|c|c|c|c|c|c|c|c|}
\hline \multirow{2}{*}{$\begin{array}{c}\text { Otvor sita, } \\
\mathbf{m m}\end{array}$} & \multicolumn{3}{|c|}{$\mathbf{3}$ min } & \multicolumn{3}{c|}{$\mathbf{5}$ min } & \multicolumn{3}{c|}{7 min } & \multicolumn{3}{c|}{$\mathbf{9}$ min } \\
\cline { 2 - 13 } & $\mathbf{m} \%$ & $\mathbf{D} \%$ & $\mathbf{R} \%$ & $\mathbf{m} \%$ & $\mathbf{D} \%$ & $\mathbf{R} \%$ & $\mathbf{m} \%$ & $\mathbf{D} \%$ & $\mathbf{R} \%$ & $\mathbf{m} \%$ & $\mathbf{D} \%$ & $\mathbf{R} \%$ \\
\hline$-0,600+0,425$ & 2,0 & 100,0 & 2,0 & & & & & & & & & \\
\hline$-0,425+0,300$ & 6,4 & 98,0 & 8,4 & & & & & & & & & \\
\hline$-0,300+0,212$ & 10,0 & 91,6 & 18,4 & 3,4 & 100,0 & 3,4 & & & & & & \\
\hline$-0,212+0,150$ & 11,4 & 81,6 & 29,8 & 7,6 & 96,6 & 11,0 & 4,8 & 100,0 & 4,80 & 2,6 & 100,0 & 2,6 \\
\hline$-0,150+0,106$ & 10,0 & 70,2 & 39,8 & 10,4 & 89,0 & 21,4 & 9,4 & 95,2 & 14,2 & 5,8 & 97,4 & 8,4 \\
\hline$-0,106+0,075$ & 6,8 & 60,2 & 46,6 & 10,6 & 78,6 & 32,0 & 11,2 & 85,8 & 25,4 & 10,6 & 91,6 & 19,0 \\
\hline$-0,075+0,053$ & 5,4 & 53,4 & 52,0 & 8,4 & 68,0 & 40,4 & 9,8 & 74,6 & 35,2 & 10,4 & 81,0 & 29,4 \\
\hline$-0,053+0,038$ & 6,0 & 48,0 & 58,0 & 7,0 & 59,6 & 47,4 & 8,6 & 64,8 & 43,8 & 10,0 & 70,6 & 39,4 \\
\hline$-0,038+0$ & 42,0 & 42,0 & 100,0 & 52,6 & 52,6 & 100,0 & 56,2 & 56,2 & 100,0 & 60,6 & 60,6 & 100,0 \\
\hline
\end{tabular}

Na slici 1 prikazana je kinetika mlevenja.

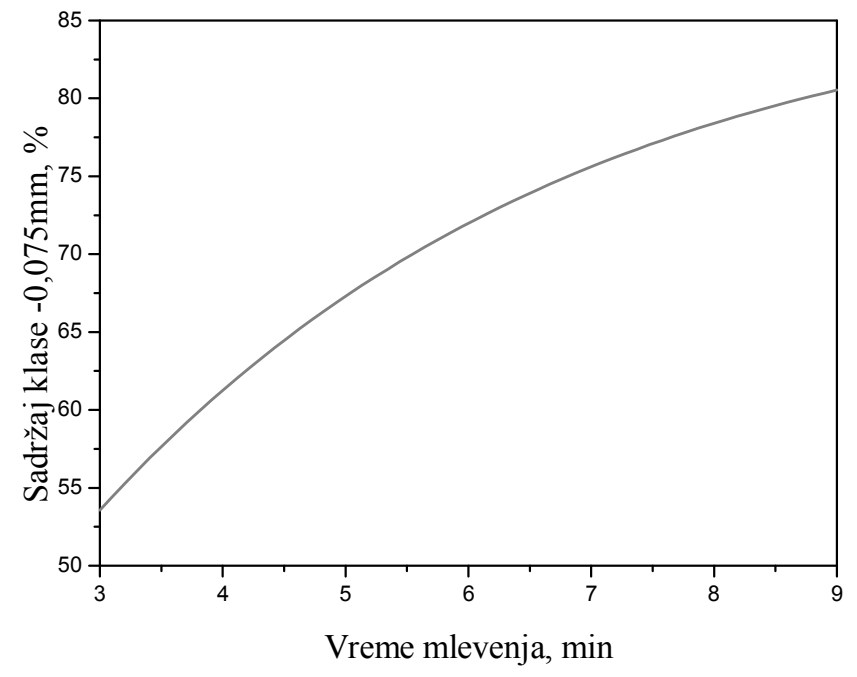

Sl. 1. Kinetika mlevenja

\section{Ispitivanje uticaja finoće mlevenja na rezultate osnovnog flotiranja}

Uticaj finoće mlevenja na rezultate flotacijskoj mašini, u komori zapremine osnovnog flotiranja ispitan je mlevenjem, 2,4 1, sa 820 g uzorka. Proizvodi koncena zatim flotiranjem $u$ Denver D-12 tracije su hemijski analizirani, a zatim je 
za svaki eksperiment računat bilans koncentracije. Izvedena je serija eksperimenata koja je obuhvatila finoću mlevenja od 55-80 \% -0,075 mm. Eksperimenti su izvedeni prema šemi prikazanoj na slici 2 .
Dobijeni rezultati koncentracije prikazani su u tablici 4. Na osnovu rezultata iz tablice 4. urađena je zavisnost iskorišćenja i sadržaja bakra u koncentratu od od finoće mlevenja i prikazana na slici 3 .

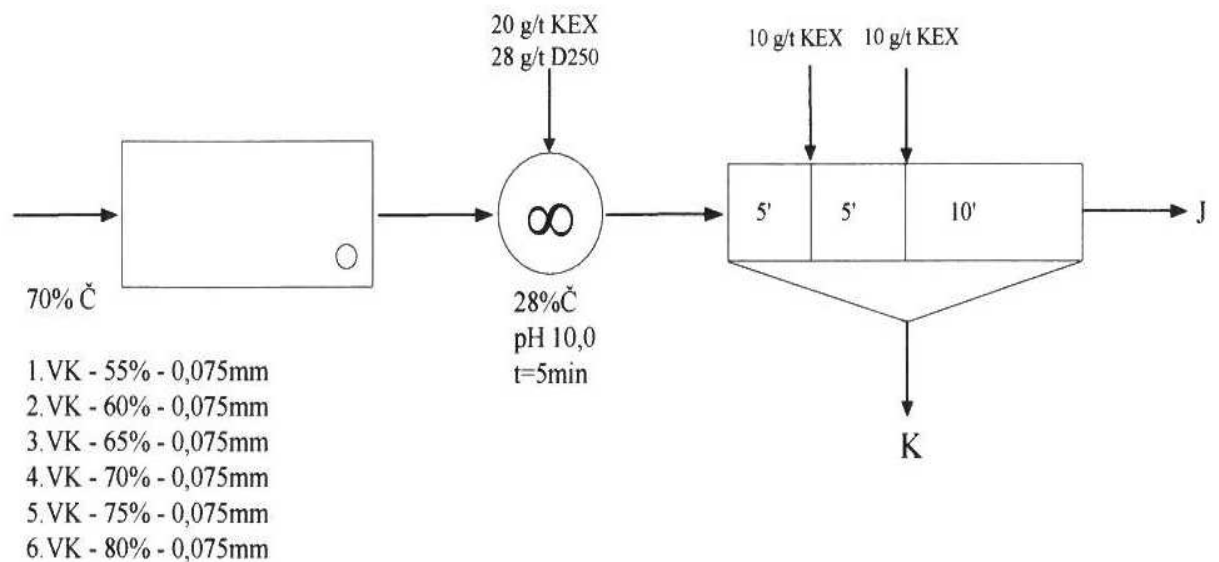

S1. 2. Šema ispitivanja uticaja finoće mlevenja

Tabela 4. Rezultati osnovnog flotiranja minerala bakra

\begin{tabular}{|c|c|c|c|c|c|}
\hline \multirow{2}{*}{ PRODUKT } & \multirow{2}{*}{$\begin{array}{c}\text { MASA } \\
\text { m, \% }\end{array}$} & \multicolumn{2}{|c|}{ SADRŽAJ } & \multicolumn{2}{|c|}{ RASPODELA } \\
\hline & & $\mathrm{Cu}, \%$ & $\mathrm{~S}, \%$ & $\mathrm{Cu}, \%$ & $\mathrm{~S}, \%$ \\
\hline \multicolumn{6}{|c|}{ Uzorak 1. VK $55 \%$ - 0,075 mm } \\
\hline $\mathrm{U}$ & 100,00 & 0,1843 & 3,5096 & 100,00 & 100,00 \\
\hline K & 10,49 & 1,50 & 31,75 & 85,40 & 94,90 \\
\hline $\mathrm{J}$ & 89,51 & 0,03 & 0,2 & 14,60 & 5,10 \\
\hline \multicolumn{6}{|c|}{ Uzorak 2. VK $60 \%-0,075 \mathrm{~mm}$} \\
\hline $\mathrm{U}$ & 100,00 & 0,2169 & 2,9674 & 100,00 & 100,00 \\
\hline $\mathrm{K}$ & 10,93 & 1,74 & 25,52 & 87,69 & 94,00 \\
\hline $\mathrm{J}$ & 89,07 & 0,03 & 0,2 & 12,31 & 6,00 \\
\hline \multicolumn{6}{|c|}{ Uzorak 3. VK 65\% - 0,075 mm } \\
\hline $\mathrm{U}$ & 100,00 & 0,2154 & 3,1449 & 100,00 & 100,00 \\
\hline $\mathrm{K}$ & 11,99 & 1,65 & 23,44 & 91,83 & 89,37 \\
\hline $\mathrm{J}$ & 88,01 & 0,02 & 0,38 & 8,17 & 10,63 \\
\hline
\end{tabular}




\begin{tabular}{|c|c|c|c|c|c|}
\hline \multicolumn{7}{|c|}{ Uzorak 4. VK 70\%- 0,075 mm } \\
\hline $\mathrm{U}$ & 100,00 & 0,2123 & 2,8124 & 100,00 & 100,00 \\
\hline $\mathrm{K}$ & 11,72 & 1,66 & 22,49 & 91,66 & 93,72 \\
\hline $\mathrm{J}$ & 88,28 & 0,02 & 0,2 & 8,34 & 6,28 \\
\hline \multicolumn{7}{|c|}{ Uzorak $5 . V K 75 \%-0,075 \mathrm{~mm}$} \\
\hline $\mathrm{U}$ & 100,00 & 0,2175 & 3,1000 & 100,00 & 100,00 \\
\hline $\mathrm{K}$ & 11,90 & 1,68 & 24,57 & 91,91 & 94,32 \\
\hline $\mathrm{J}$ & 88,10 & 0,02 & 0,2 & 8,09 & 5,68 \\
\hline \multicolumn{7}{|c|}{ Uzorak $6 . V K 80 \%-0,075 \mathrm{~mm}$} \\
\hline $\mathrm{U}$ & 100,00 & 0,2319 & 3,6072 & 100,00 & 100,00 \\
\hline $\mathrm{K}$ & 12,61 & 1,70 & 27,22 & 92,45 & 95,15 \\
\hline $\mathrm{J}$ & 87,39 & 0,02 & 0,2 & 7,55 & 4,85 \\
\hline
\end{tabular}

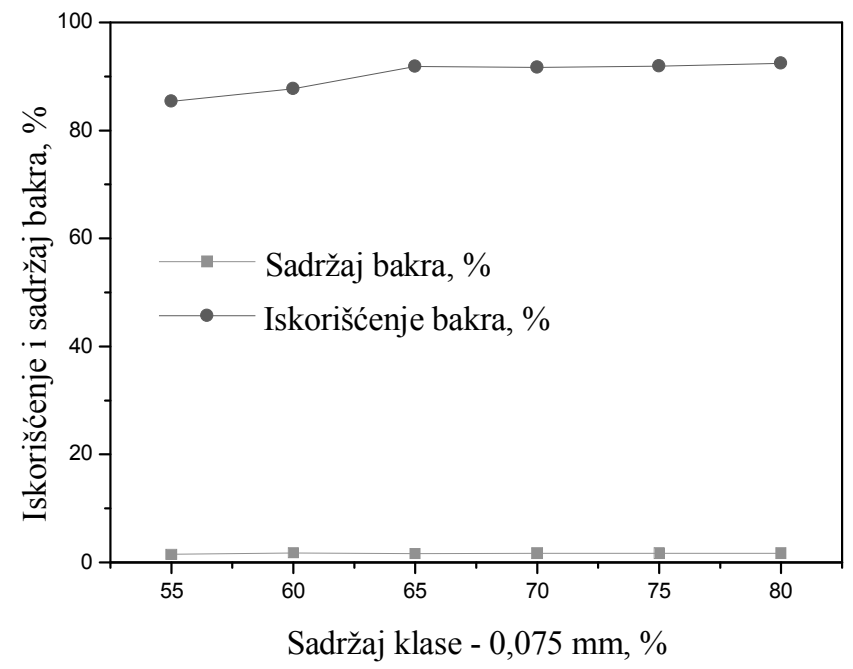

SI. 3. Zavisnost iskorišćenja i sadržaja bakra u osnovnom koncentratu od finoće mlevenja

\section{Domeljavanje}

Za ispitivanje domeljavanja korišćen je laboratorijski elipsoidni mlin sa kuglama zapremine 15,2 1, sa masom šarže kugli na

početku ispitivanja od $3,755 \mathrm{~kg}$. Za svaki eksperiment domeljavanja, bilo je potrebno samleti po četiri uzorka mase $820 \mathrm{~g}$. Nakon 
toga, izvršena su po dva osnovna flotiranja (pri čemu je masa uzoraka u svakom osnovnom flotiranju iznosila $1.640 \mathrm{~g}$ ) $\mathrm{u}$ flotacijskoj mašini Denver D-12 u komori zapremine 4,6 1 prema istim uslovima, a zatim je dobijeni osnovni koncentrat domeljavan u mlinu sa kuglama u različitim vremenskim intervalima. Proizvod domeljavanja je jednom prečišćen u flotacijskoj mašini Denver D-12 u komori od 2,4 1, a proizvodi koncentracije hemijski analizirani nakon čega je računat bilans koncentracije.

\section{Ispitivanje kinetike domeljavanja}

Šema prema kojoj je ispitana kinetika domeljavanja prikazana je na slici 4. Rezultati granulometrijske analize proizvoda domeljavanja prikazani su u tablici 6, a kinetika domeljavanja na slici 5 .

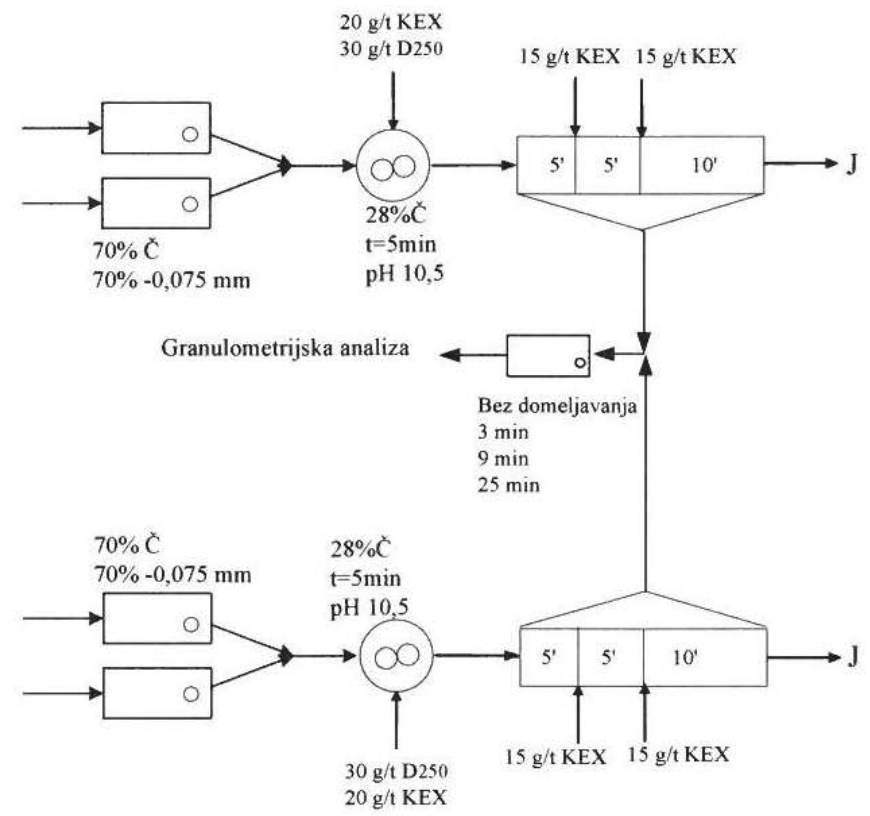

Sl. 4. Šema ispitivanja kinetike domeljavanja

Tabela 6. Granulometrijski sastav proizvoda domeljavanja za različita vremena domeljavanja

\begin{tabular}{|c|c|c|c|c|c|c|c|c|}
\hline $\begin{array}{c}\text { Otvor sita, } \\
\text { mm }\end{array}$ & \multicolumn{2}{|c|}{ Bez dom. } & \multicolumn{2}{c|}{ 3 min. } & \multicolumn{2}{c|}{ 9 min. } & \multicolumn{2}{c|}{ 25 min. } \\
\cline { 2 - 9 } & $\mathbf{m} \%$ & D\% & m \% & D \% & m \% & D \% & m \% & D \% \\
\hline$-0,300+0,212$ & 2,00 & 100,00 & & & & & & \\
\hline$-0,212+0,150$ & 6,67 & 98,00 & 2,57 & 100,00 & & & & \\
\hline$-0,150+0,106$ & 11,89 & 91,33 & 7,38 & 97,43 & 3,32 & 100,00 & 0,86 & 100,00 \\
\hline$-0,106+0,075$ & 11,22 & 79,44 & 10,81 & 90,05 & 7,00 & 96,68 & 3,86 & 99,14 \\
\hline$-0,075+0,053$ & 8,22 & 68,22 & 9,81 & 79,24 & 9,84 & 89,68 & 6,72 & 95,28 \\
\hline$-0,053+0,038$ & 7,00 & 60,00 & 8,62 & 69,43 & 9,84 & 79,84 & 8,72 & 88,56 \\
\hline$-0,038+0$ & 53,00 & 53,00 & 60,81 & 60,81 & 70,00 & 70,00 & 79,84 & 79,84 \\
\hline
\end{tabular}




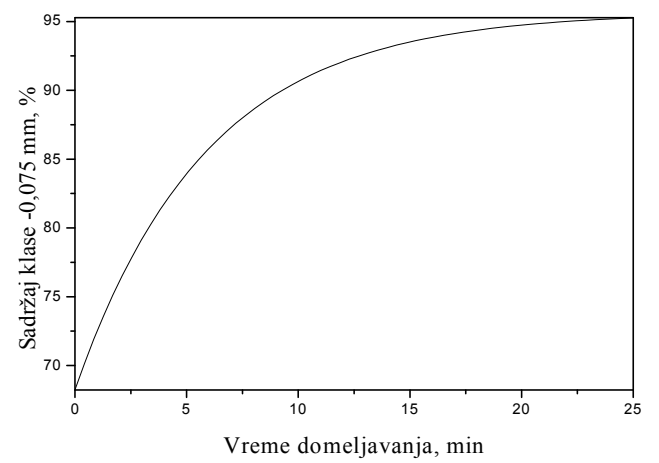

SI. 5. Kinetika domeljavanja

Uticaj finoće domeljavanja na rezultate flotacijske koncentracije

Uticaj finoće domeljavanja na rezultate flotacijske koncentracije ispitan je kroz četiri eksperimenta, a šema prema kojoj su eksperimenti izvedeni prikazana je na slici 6. Rezultati uticaja domeljavanja na preči- šćavanje koncentrata bakra prikazani su u tablici 7 i na slici 7 prikazana je zavisnost iskorišćenja i sadržaja bakra u prečišćenom koncentratu bakra od finoće domeljavanja.

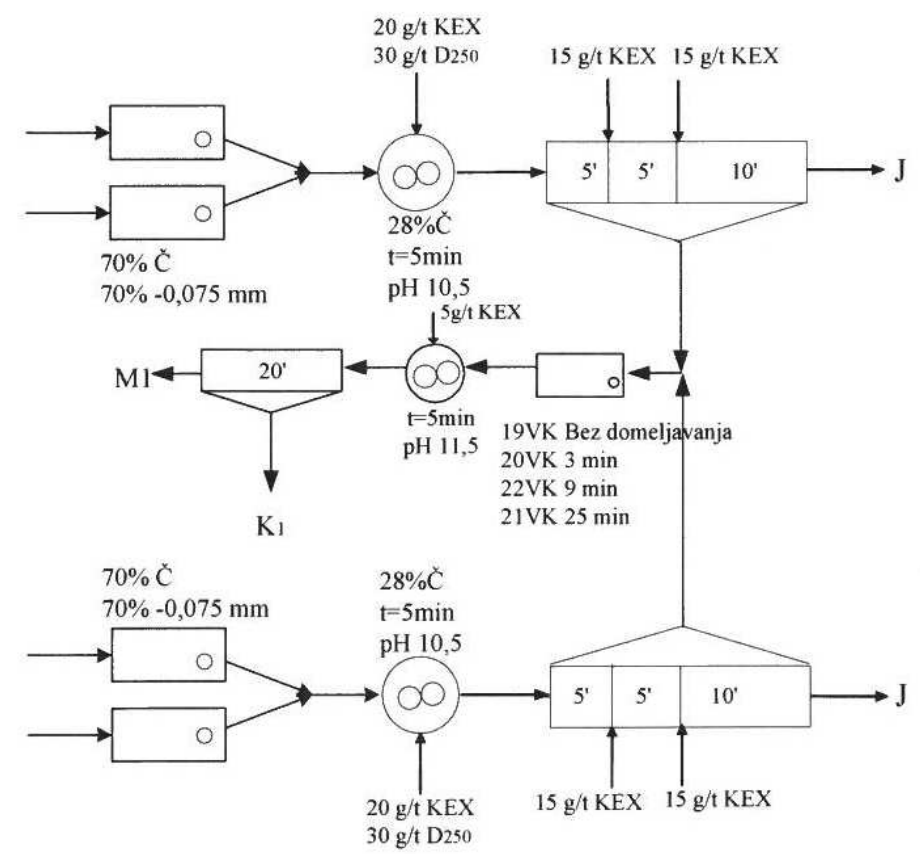

Sl. 6. Šema ispitivanja uticaja finoće domeljavanja na rezultate flotiranja 
Tabela 7. Uticaj finoće domeljavanja na rezultate prečišćavanja koncentrata bakra

\begin{tabular}{|c|c|c|c|c|c|c|c|c|c|}
\hline \multirow[b]{2}{*}{ PROD. } & \multirow{2}{*}{$\begin{array}{c}\text { MASA } \\
\text { m, \% }\end{array}$} & \multicolumn{4}{|c|}{ SADRŽAJ } & \multicolumn{4}{|c|}{ RASPODELA } \\
\hline & & $\begin{array}{c}\mathrm{Cu}, \\
\%\end{array}$ & $\mathrm{~S}, \%$ & $\begin{array}{c}\mathbf{A u}, \\
\mathrm{g} / \mathrm{t}\end{array}$ & $\begin{array}{l}\mathrm{Ag}, \\
\mathrm{g} / \mathrm{t}\end{array}$ & $\begin{array}{c}\mathrm{Cu}, \\
\%\end{array}$ & $\mathrm{~S}, \%$ & $\begin{array}{c}\mathbf{A u}, \\
\mathrm{g} / \mathrm{t}\end{array}$ & $\begin{array}{c}\text { Ag, } \\
\text { g/t }\end{array}$ \\
\hline \multicolumn{10}{|c|}{ Uzorak 19.VK-68,22\%-0,075 mm; Bez domeljavanja } \\
\hline $\mathrm{U}$ & 100,00 & 0,1855 & 2,8081 & 0,073 & 0,80 & 100,00 & 100,00 & 100,00 & 100,00 \\
\hline $\mathrm{J}$ & 90,36 & 0,010 & 0,27 & 0,016 & 0,713 & 4,85 & 8,69 & 19,25 & 80,58 \\
\hline Ko & 9,64 & 1,83 & 26,60 & 0,612 & 1,612 & 95,15 & 91,31 & 80,75 & 19,42 \\
\hline $\mathrm{M}_{1}$ & 7,49 & 0,14 & 26,89 & 0,5 & 1,50 & 5,66 & 71,71 & 51,30 & 14,04 \\
\hline $\mathrm{K}_{1}$ & 2,15 & 7,72 & 25,60 & 1,0 & 2,00 & 89,49 & 19,60 & 29,45 & 5,38 \\
\hline \multicolumn{10}{|c|}{ Uzorak 20 VK - 79,24\%-0,075 mm; 3 minuta } \\
\hline $\mathrm{U}$ & 100,00 & 0,1864 & 3,1504 & 0,080 & 0,80 & 100,00 & 100,00 & 100,00 & 100,00 \\
\hline $\mathrm{J}$ & 89,44 & 0,012 & 0,2 & 0,019 & 0,671 & 5,74 & 5,68 & 21,24 & 75,05 \\
\hline Ko & 10,56 & 1,66 & 28,14 & 0,597 & 1,89 & 94,26 & & 78,86 & 24,95 \\
\hline $\mathrm{M}_{1}$ & & 0,06 & 28,99 & 0,384 & 1,70 & 2,74 & & 41,07 & 18,17 \\
\hline $\mathrm{K}_{1}$ & 2,01 & 8,49 & 24,52 & 1,50 & 2,70 & 91,52 & 15,65 & 37,69 & 6,78 \\
\hline \multicolumn{10}{|c|}{ Uzorak 22 VK - 89,68\%-0,075 mm; 9 minuta } \\
\hline $\mathrm{U}$ & 100,00 & 0,1988 & 3,0517 & 0,073 & 0,80 & 100,00 & 100,00 & 100,00 & 100,00 \\
\hline $\mathrm{J}$ & 90,69 & 0,012 & 0,36 & 0,020 & 0,68 & 5,48 & 10,70 & 24,85 & 77,09 \\
\hline Ko & 9,31 & 2,02 & 29,27 & 0,589 & 1,969 & 94,52 & 89,30 & 75,15 & 22,91 \\
\hline $\mathrm{M}_{1}$ & 7,78 & 0,14 & 29,89 & 0,414 & 1,80 & 5,48 & 76,20 & 44,16 & 17,50 \\
\hline $\mathrm{K}_{1}$ & 1,53 & 11,57 & 26,13 & 1,478 & 2,828 & 89,03 & 13,10 & 30,99 & 5,41 \\
\hline \multicolumn{10}{|c|}{ Uzorak $21-95,28 \%-0,075 \mathrm{~mm} ; 25$ minuta } \\
\hline $\mathrm{U}$ & 100,00 & 0,1980 & 2,8367 & 0,073 & 0,80 & 100,00 & 100,00 & 100,00 & 100,00 \\
\hline $\mathrm{J}$ & 90,35 & 0,012 & 0,2 & 0,017 & 0,706 & 5,45 & 6,37 & 21,04 & 79,73 \\
\hline Ko & 9,65 & 1,94 & 27,52 & 0,597 & 1,681 & 94,55 & 93,63 & 78,96 & 20,27 \\
\hline$\overline{M_{1}}$ & 8,31 & 0,15 & 27,92 & 0,403 & 1,50 & 6,31 & 81,79 & 45,92 & 15,58 \\
\hline $\mathrm{K}_{1}$ & 1,34 & 13,04 & 25,06 & 1,80 & 2,80 & 88,23 & 11,84 & 33,04 & 4,69 \\
\hline
\end{tabular}

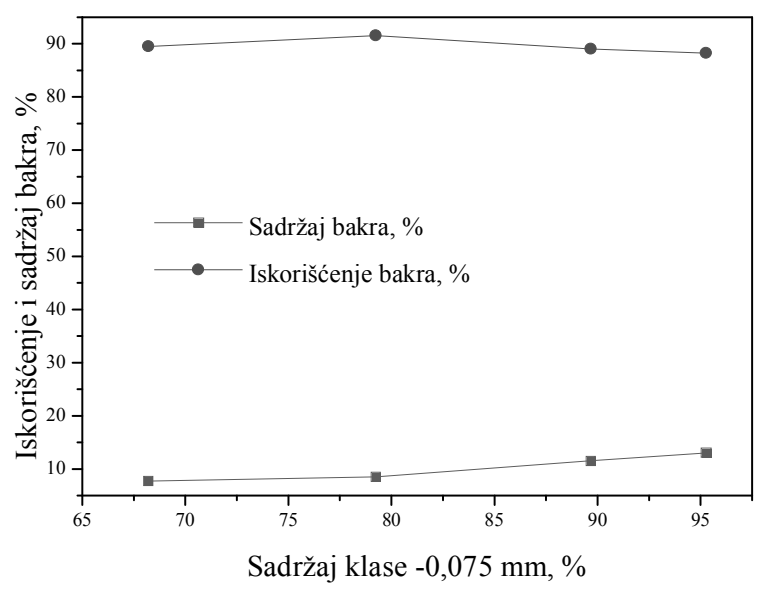

SI. 7. Uticaj finoće domeljavanja na rezultate koncentracije 


\section{ZAKLJUČAK}

Optimalna finoća mlevenja u procesu flotacijske koncentracije na rudi ležišta Veliki Krivelj određena je na bazi laboratorijskih ispitivanja koja su prethodila projektovanju pogona i otvaranju rudnika. Danas, gotovo 30 godina od otvaranja rudnika, finoća mlevenja $u$ pogonu održava se kao i na početku proizvodnje. Da bi se utvrdilo da li ovaj važan parametar odgovara rudi koja se danas prerađuje izvršena su ispitivanja na uzorku rude izuzetom iz proizvodnje. Uzorak rude Veliki Krivelj je označen oznakom VK, skraćen i usitnjen do krupnoće $-3,35 \mathrm{~mm}$. Izuzeti su uzorci za hemijsku analizu i eksperimente flotacijske koncentracije i na njima izvršena ispitivanja.

Srednji sadržaj važnijih elemenata u uzorku, izračunato iz hemijske analize je: 0,2133\% Cu; 3,0067 \% S; 0,0733 g/t Au; $0,5667 \mathrm{~g} / \mathrm{t}$ Ag. Srednji sadržaj važnijih elemenata u uzorku VK, kada se izračuna preko proizvoda koncentracije svih eksperimenata je: $0,2095 \% \mathrm{Cu} ; 3,1998 \% \mathrm{~S}$; $0,0709 \mathrm{~g} / \mathrm{t} \mathrm{Au} ; 0,6614 \mathrm{~g} / \mathrm{t} \mathrm{Ag}$. Gustina uzorka VK iznosila je $2.820 \mathrm{~kg} / \mathrm{m}^{3}$, prirodna $\mathrm{pH}$ 7,20. Tokom laboratorijskih tehnoloških ispitivanja na uzorku VK došlo se do sledećih zaključaka:

- Minimalna finoća mlevenja koju treba ostvariti na uzorku VK, sa aspekta tehnoloških reziltata je $65 \%$ $-0,075 \mathrm{~mm}$

- Domeljavanje osnovnog koncentrata nema velikog uticaja na iskorišćenje bakra u koncentratu prvog prečišćavanja, ali se sadržaj bakra u koncentratu povećava. Rezultati ukazuju da je domeljavanje osnov- nog koncentrata poželjno i to do 80$90 \%-0,075 \mathrm{~mm}$. Ne treba ići preko $90 \%-0,075 \mathrm{~mm}$ jer u tom slučaju opada iskorišćenje bakra

Rezultati laboratorijskih eksperimenata pokazuju da je minimalna finoća mlevenja koju treba ostvariti na uzorku VK 65\% $0,075 \mathrm{~mm}$. Time se povećava iskorišćenje bakra u osnovnom koncentratu za oko $4 \%$. Dalje povećanje finoće mlevenja dovelo bi do povećanja iskorišćenja bakra za još oko $1 \%$.

Autori ispitivanja smatraju da bi odgovorni u RTB Bor trebali da razmotre mogućnost povećanja finoće mlevenja rude ležišta Veliki Krivelj, jer će to sigurno imati pozitivne finansijske efekte. Kompleksnija ekonomska analiza bi mogla tačno da pokaže do koje finoće treba ići, jer je to kompromis između nekoliko bitnih stavki u proizvodnji:

- povećanja potrošnje energije na finije mlevenje,

- povećanja količine bakra u koncentratu sa povećanjem finoće mlevenja i

- smanjenja kapaciteta mlinskih sekcija, jer je samo tako u datim uslovima moguće finije mlevenje.

\section{LITERATURA}

[1] Mitrović Z., Jovanović R., Sto godina borskog rudarstva 1903-2003, Sedamdeset pet godina basenskih flotacija 1929-2003., Rudarsko-topioničarski basen Bor, Megatrend univerzitet Beograd, 2007.

[2] Bugarin M., Maksimović M., Ljubojev V., Copper and Gold Reserves in the Exploratory - Mining Field of RTB 
Bor; Mining Engineering, Mining and Metallurgy Institute Bor, 3/2012, pp. 9-16.

[3] Barry W. A., Napier-Munn T., Wills' Mineral Processing Technology, Elsevier Science\&Technology Books, 2006.
[4] Gaudin A. M., Flotation, McGraw-Hill Book Company, Inc., New York, Toronto, London, 1957.

[5] Bulatovic S. M., Handbook og Flotation Reagents, Volume 1, Elsevier Science \& Technology Books, 2007. 